\title{
Comment on "Molecular Selectivity in Aquaporin Channels Studied by the 3D-RISM Theory"
}

\author{
Jochen S. $\mathrm{Hub}^{\dagger}$ and Bert L. de Groot ${ }^{*,+}$ \\ ${ }^{\dagger}$ Department of Cell and Molecular Biology, Uppsala University, Box 596, 75124 Uppsala, Sweden \\ ${ }^{\ddagger}$ Computational Biomolecular Dynamics Group, Max-Planck-Institute for Biophysical Chemistry, Am Fassberg 11, 37077 Göttingen, \\ Germany
}

Tn a recent article, Phongphanphanee et al. present a study on the molecular selectivity of the water channel aquaporin-1 (AQP1) and of the aquaglyceroporin GlpF from E. coli. ${ }^{1}$ The authors apply the three-dimensional reference interaction site model (3D-RISM) theory, in order to compute distribution functions of a number of solutes within the channels, and, in a second step, derive potentials of mean force (PMFs) from these distribution functions. Accordingly, Phongphanphanee et al. arrive at PMFs for the permeation of a number of solutes across AQP1 and GlpF including $\mathrm{H}_{2} \mathrm{O}, \mathrm{CO}_{2}, \mathrm{NH}_{3}$, urea, and glycerol. In a previous study, ${ }^{2}$ we have also computed PMFs for the permeation of these solutes across AQP1 and GlpF, however using a different computational approach. We applied molecular dynamics (MD) simulations, using the technique of umbrella sampling simulation.

Several of the PMFs reported by Phongphanphanee et al. are (nearly) barrierless, including the PMFs for $\mathrm{H}_{2} \mathrm{O}$ and $\mathrm{NH}_{3}$ permeation through AQP1 as well as all PMFs for solute permeation across GlpF. ${ }^{1}$ In contrast, the respective PMFs presented by us display a barrier of $\sim 12 \mathrm{~kJ} / \mathrm{mol}$. Phongphanphanee et al. suggest that their PMFs are in agreement with experiments, whereas the authors conclude from the barriers in our PMFs that our results are in "sharp contrast" to or "entirely opposite" of experimental results. The authors continue to argue that our MD simulations "fail to describe the experimental phenomena even in a qualitative manner" and that our PMFs would imply that AQP1 and GlpF are "not permeable by those ligands". With this Comment, we explain why we disagree with these statements and show that the PMFs by Phongphanphanee and coworkers correspond to an undefined channel concentration, rendering a direct comparison to experiments problematic. As detailed below, the apparent discrepancy is based on the fact that our permeation PMFs refer to a well-defined membrane area per channel, whereas Phongphanphanee et al. disregard any such reference area.

PMFs for full permeation events across channels describe the energetics for the displacement of the solute from one bulk water regime through the channel and into the other bulk water regime (Figure 1A). For such PMFs, the definition of the free energy for the bulk states needs to be taken into account, where the solute is not confined in the $x-y$ plane (membrane plane) by interaction with the channel. If the solute could diffuse in an infite $x-y$ plane, the solute could adopt an infinte number of states, leading to undefined free energies. At a finite channel concentration, as occurs under experimental and physiological conditions, this effect leads to a well-defined entropic penalty for a molecule entering the channel that is directly dependent on membrane area per channel.
A common approach to ensure well-defined bulk free energies and to allow the comparison to experiment is to confine the solute within an $x-y$ area around the channel. We will refer to that area as the reference area $A_{\text {ref }}$ of the PMF. $A_{\text {ref }}$ can be chosen freely; it must only be known and be reported with the PMF as it defines the bulk level in the PMF and the channel concentration in the membrane. Note that the PMF $G(z)$ referring to $A_{\text {ref }}$ can be referred to a different reference area $A_{\text {ref }}^{\prime}$ by applying a correction of $\Delta G_{\text {ref }}=-k_{\mathrm{B}} T \ln \left(A_{\text {ref }}^{\prime} / A_{\text {ref }}\right)$ in the entrance and exit regions of the pore. Here, $\Delta G_{\text {ref }}$ accounts for the different entropies for a solute confined in $A_{\text {ref }}^{\prime}$ as compared to a solute confined in $A_{\text {ref, }}$ and $k_{\mathrm{B}}$ and $T$ denote the Boltzmann constant and the temperature, respectively. For a more quantitative definition, we refer to the excellent overview by Allen et al. ${ }^{3}$

Although $A_{\text {ref }}$ can be chosen freely, some choices for $A_{\text {ref }}$ are useful to rationalize the findings of the PMF. For instance, given a specific density of channels in the membrane, a natural choice for $A_{\text {ref }}$ would be the membrane area per channel. Because the density of channels depends on the specific experiment and is frequently unknown, and as clearly stated in our publication, ${ }^{2}$ we chose as the reference area the cross section area occupied by one aquaporin monomer in the lipid bilayer, in the following referred to as $A_{\text {mono }}$. For AQP1 and GlpF, $A_{\text {mono }}$ equals approximately 1030 and $1070 \AA^{2}$, respectively. As shown below, these areas lead to barrier heights of at at least $12 \mathrm{~kJ} / \mathrm{mol}$, which reflect the entropic cost for a molecule to be confined to the narrow channel. ${ }^{2}$ Hence, our PMFs refer to the closest possible packing of channels, corresponding to a two-dimensional aquaporin crystal. This specific choice for $A_{\text {ref }}$ is useful to compare the channel PMFs to PMFs for solute permeation across the lipid bilayer; if the channel PMF exhibits a lower barrier than the lipid bilayer PMF, insertion of the channel into such a membrane would increase the solute flux. In contrast, no such increased flux is expected for a higher barrier in the channel as compared to the lipid bilayer. Noteworthy, our PMFs can be translated to a specific experimental density of channels using the procedure pointed out in the previous paragraph.

In contrast, no reference area is reported for the PMFs by Phongphanphanee et al. ${ }^{1}$ Consequently, these PMFs may provide information on solute distribution within the pore, but it is impossible for the reader to relate these PMFs to any experimental density of channels and hence to validate the PMFs quantitatively. In addition, the barrier heights in the PMFs by Phongphanphanee et al. ${ }^{1}$ cannot be compared to our PMFs. ${ }^{2}$

Received: $\quad$ March 9, 2011

Published: June 06, 2011 


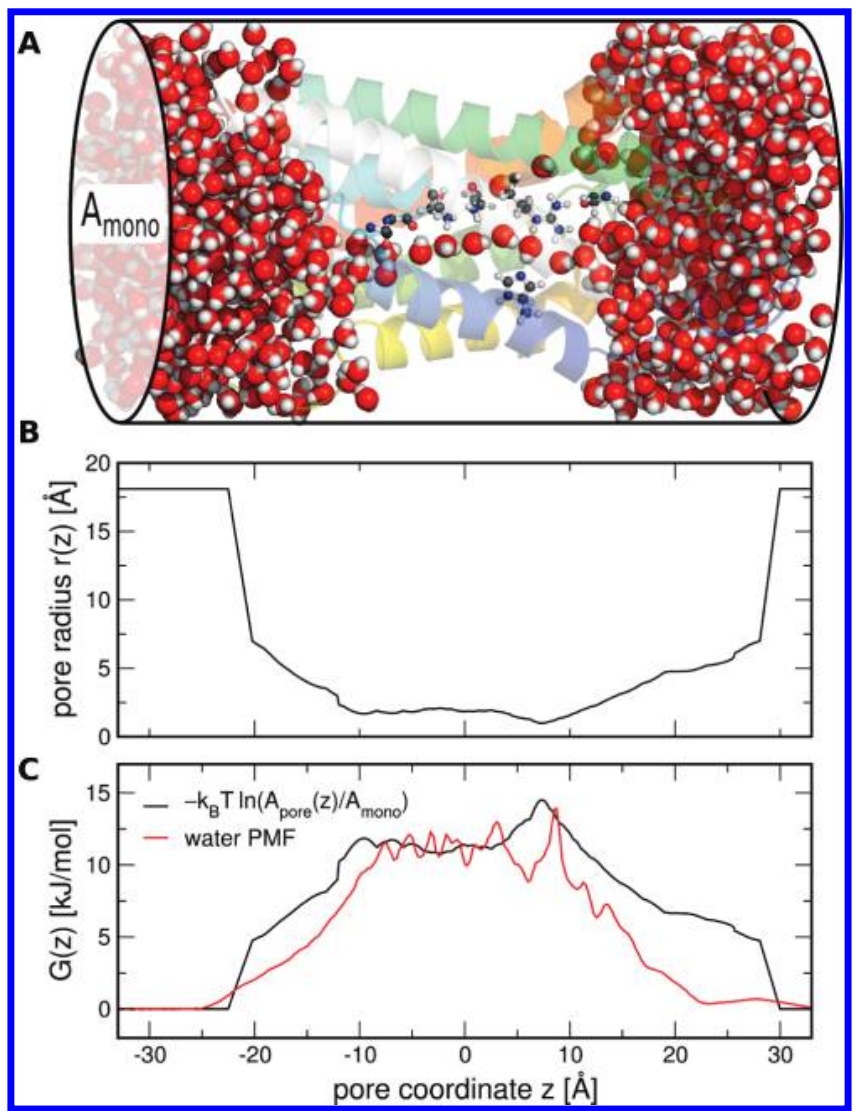

Figure 1. (A) Simulation snapshot of an aquaporin-1 (AQP1) monomer (cartoon representation), filled by a single file of water (red and white spheres). Bulk water within a cylinder of cross section area $A_{\text {mono }}=$ $1030 \AA^{2}$ is shown, where $A_{\text {mono }}$ denotes the cross section area occupied by an AQP1 monomer in the lipid bilayer. (B) Pore radius $r(z)$ of the AQP1 as derived by HOLE. ${ }^{4}$ In the two bulk water regimes, the radius was set to $\left(A_{\text {mono }} / \pi\right)^{1 / 2}$. (C, black) Reduced conformational freedom of water molecules in the pore $(|z|<10 \AA)$ as compared to the water molecules in the bulk $(|z|>20 \AA)$. The cross section area of the pore $A_{\text {pore }}(z)=\pi r(z)^{2}$ yields an entropic contribution to the PMF of $T \Delta S(z)=-k_{\mathrm{B}} T \ln \left(A_{\text {pore }}(z) / A_{\text {mono }}\right)$. (C, red) The PMF for water permeation across AQP1 derived by counting water occupancies along the pore in a $20 \mathrm{~ns}$ molecular dynamics simulation. ${ }^{2}$ The PMF resembles the entropic profile (black), which was based on the cross section only. Thus, a barrier of $\sim 14 \mathrm{~kJ} / \mathrm{mol}$ in the PMF mainly reflects the reduced radius of the pore (as compared to the radius of the cylinder), and it does not indicate an empty channel.

Phongphanphanee et al. conclude from the fact that our PMFs exhibit barriers $>12 \mathrm{~kJ} / \mathrm{mol}$ that our PMFs would indicate nonconducting empty channels. This is a wrong assessment of our PMFs, as illustrated for the example of water permeation through AQP1 in Figure 1. Figure 1A shows an AQP1 monomer, filled by a single file of water and solvated by two bulk water compartments. Here, only water molecules within a cylinder of cross section area $A_{\text {mono }}$ are shown. Figure $1 \mathrm{~B}$ presents the radius $r(z)$ of the pore as computed by HOLE, ${ }^{4}$ where the radius in the bulk is set to the radius of the cylinder. Hence, the $r(z)$ curve reflects the geometric freedom in the $x-y$ plane of water molecules that are confined to the cross section area $A_{\text {mono. The radius }} r(z)$ can be translated into an entropic profile via $T \Delta S(z)=-k_{\mathrm{B}} T \ln \left(\pi r(z)^{2} /\right.$ $A_{\text {mono }}$ ) (Figure 1C, black). For comparison, Figure $1 \mathrm{C}$ also presents the PMF for water permeation through AQP1, which has been computed based on counting water occupancies in a $20 \mathrm{~ns}$ simulation. ${ }^{2}$ Remarkably, the PMF exhibits a similar barrier height compared to the entropic profile (black), demonstrating that the barrier of $14 \mathrm{~kJ} / \mathrm{mol}$ mainly reflects the narrowing of the pore as compared to the bulk regions. In fact, a barrier of $14 \mathrm{~kJ} / \mathrm{mol}$ refers to a permanently filled and highly conducting water channel, as shown in Figure 1A, and does by no means "exclude any possibility of the water molecule existing in the channels", as incorrectly concluded by Phongphanphanee et al. ${ }^{1}$

As the undefined bulk level in the PMFs of Phongphanphanee et al. ${ }^{1}$ exclude a quantitative assessment of the absolute barrier heights, we instead focus on relative barrier heights for two examples.

First, we consider ammonia permeation through AQP1 and GlpF. We found barriers of 18 and $12.5 \mathrm{~kJ} / \mathrm{mol}$, respectively, suggesting that GlpF conducts ammonia $\sim 10$ times more efficiently than AQP1. As discussed in detail in our original publication, ${ }^{2}$ these results are in line with experiments by Holm, ${ }^{5}$ Beitz, ${ }^{6}$ Saparov, ${ }^{7}$ and co-workers, who found increased ammonia flux after expression of aquaglyceroporins compared to control measurements, whereas AQP1 did not increase the ammonia flux. In contrast, Phongphanphanee et al. reported barrierless ammonia permeation for GlpF and a low barrier of $2.5 \mathrm{~kJ} / \mathrm{mol}$ for AQP1. ${ }^{1}$ This would indicate that AQP1 and GlpF are about equally efficient ammonia channels, in disagreement with the experimental result. ${ }^{5,6}$

As a second example, we consider glycerol and urea permeation through GlpF. Our PMFs exhibit barriers of 13.5 and $29 \mathrm{~kJ} /$ mol for glycerol and urea, respectively. These barriers suggest that GlpF is permeated by glycerol 500 times more efficiently than by urea. Together with a 7 -fold higher lipid bilayer permeability for glycerol as compared to urea (estimated by applying Overton's rule and inserting the hexadecane/water partition coefficients of $2.0 \times 10^{-6}$ and $2.8 \times 10^{-7}$ for glycerol and urea, respectively ${ }^{8}$ ), our barriers imply that the glycerol flux increases $\sim 70$ fold more strongly than that of urea upon reconstitution of GlpF into the bilayer. These numbers agree favorably with Borgnia and Agre, ${ }^{9}$ who found a 400 -fold increase of glycerol flux after reconstitution of GlpF into liposomes but only a 3-fold increase of urea flux (at $5{ }^{\circ} \mathrm{C}$ ). In contrast, Phongphanphanee et al. reported a nearly flat PMF for both glycerol and urea permeation across GlpF, ${ }^{1}$ in disagreement with the experiments by Borgnia and Agre. 'Despite these discrepancies, Phongphanphanee et al. erroneously conclude that their "results are in good accord with the experimental results for the case of glycerol and urea, which are known to be permeable through the channel”.

In referring to our work, Phongphanphanee et al. unfoundedly claim that the "trouble in the simulation results originates mainly from the insufficient sampling of the configuration space of the ligand molecules in the channel, and that it is caused by the choice of order parameters the authors of the paper have made in the umbrella sampling". ${ }^{1}$ No evidence is provided to support this criticism.

In fact, in order to validate the umbrella sampling procedure, we have computed the PMF for water permeation across AQP1 and GlpF using two independent approaches, (i) umbrella sampling and (ii) by counting water molecule occupancies in $20 \mathrm{~ns}$ equilibrium simulations. The two approaches agree within $3 \mathrm{~kJ} / \mathrm{mol}$, suggesting that the umbrella sampling procedure is reliable (Figure S10 of ref 2 ). In addition, we have validated the parameters of all solutes by reproducing the hexadecane/ water partition coefficient, and we have computed the statistical 
uncertainty in the PMFs using a robust bootstrapping approach, ${ }^{10}$ demonstrating that the PMFs are converged (illustrated in Figures S7 and S9 of ref 2) and that the simulation results do not suffer from insufficient sampling.

Taken together, our simulation results quantitatively agree with a large set of experimental data. In addition, the methodological procedures are backed up through a number of theoretical validation steps. We hence rate the criticism raised by Phongphanphanee et al. as unfounded. The undefined channel concentration translates into an undefined bulk level in the PMFs of Phongphanphanee et al., rendering a comparison of absolute barrier heights to experimental observations meaningless. For ammonia permeation through AQP1 and GlpF and glycerol and urea permeation through $\mathrm{GlpF}$, the relative PMF barriers of Phongphanphanee et al. are in disagreement with experimental observations.

\section{AUTHOR INFORMATION}

\section{Corresponding Author}

*E-mail: bgroot@gwdg.de.

\section{REFERENCES}

(1) Phongphanphanee, S.; Yoshida, N.; Hirata, F. I. Phvs. Chem. B 2010, 114, 7967-7973.

(2) Hub, J. S.; de Groot, B. L. Proc. Natl. Acad. Sci. U.S.A. 2008, 105, 1198-1203.

(3) Allen, T. W.; Andersen, O. S.; Roux, B. Biophys. Chem. 2006, $124,251-267$.

(4) Smart, O. S.; Neduvelil, J. G.; Wang, X.; Wallace, B. A.; Sansom, M. S. P. L. Mol. Graphics 1996, 14, 354-360.

(5) Holm, L. M.; Jahn, T. P.; Møller, A. L. B.; Schjoerring, J. K.; Ferri, D.; Klaerke, D. A.; Zeuthen, T. Pfluegers Arch. 2005, 450, 415-428.

(6) Beitz, E.; Wu, B.; Holm, L. M.; Schultz, J. E.; Zeuthen, T. Proc. Natl. Acad. Sci. U.S.A. 2006, 103, 269-274.

(7) Saparov, S. M.; Liu, K.; Agre, P.; Pohl, P. I. Biol. Chem. 2007, 282, 5296-5301.

(8) Walter, A.; Gutknecht, J. L.Membr. Biol. 1986, 90, 207-217.

(9) Borgnia, M. J.; Agre, P. Proc. Natl. Acad. Sci. U.S.A. 2001, 98, 2888-2893.

(10) Hub, J. S.; de Groot, B. L.; van der Spoel, D. L. Chem. Theory Comput. 2010, 6, 3713-3720. 